\title{
Depth-Dependent Temporal Response Properties in Core Auditory Cortex
}

\author{
G. Björn Christianson, ${ }^{1}$ Maneesh Sahani, ${ }^{2}$ and Jennifer F. Linden ${ }^{1,3}$ \\ ${ }^{1}$ UCL Ear Institute, University College London, London WC1X 8EE, United Kingdom, ${ }^{2}$ Gatsby Computational Neuroscience Unit, University College \\ London, London WC1N 3AR, United Kingdom, and '3Department of Neuroscience, Physiology and Pharmacology, University College London, London \\ WC1E 6BT, United Kingdom
}

The computational role of cortical layers within auditory cortex has proven difficult to establish. One hypothesis is that interlaminar cortical processing might be dedicated to analyzing temporal properties of sounds; if so, then there should be systematic depthdependent changes in cortical sensitivity to the temporal context in which a stimulus occurs. We recorded neural responses simultaneously across cortical depth in primary auditory cortex and anterior auditory field of CBA/Ca mice, and found systematic depth dependencies in responses to second-and-later noise bursts in slow (1-10 bursts/s) trains of noise bursts. At all depths, responses to noise bursts within a train usually decreased with increasing train rate; however, the rolloff with increasing train rate occurred at faster rates in more superficial layers. Moreover, in some recordings from mid-to-superficial layers, responses to noise bursts within a 3-4 bursts/s train were stronger than responses to noise bursts in slower trains. This non-monotonicity with train rate was especially pronounced in more superficial layers of the anterior auditory field, where responses to noise bursts within the context of a slow train were sometimes even stronger than responses to the noise burst at train onset. These findings may reflect depth dependence in suppression and recovery of cortical activity following a stimulus, which we suggest could arise from laminar differences in synaptic depression at feedforward and recurrent synapses.

\section{Introduction}

A characteristic feature of the cerebral cortex is the organization of neurons into laminae differing in cell density and morphology and in patterns of connectivity to other brain areas (deFelipe and Jones, 1988). It has long been hypothesized that these laminae play different roles in cortical computation, and that variations in response properties between the layers provide insight into the nature of those computations (Douglas and Martin, 2004). Laminar differences in response properties have been identified in visual (Hubel and Wiesel, 1962; Gilbert, 1977) and somatosensory cortex (Brumberg et al., 1999; Ahissar et al., 2001), and have inspired hypotheses about cortical computation in vision and somatosensation. However, in auditory cortex, the existence and form of laminar differences in response properties has proven controversial (Linden and Schreiner, 2003). Basic response properties, such as latency and tuning bandwidth, show different variations with cortical depth across studies, even within the same species (Abeles and Goldstein, 1970; Phillips and Irvine, 1981;

\footnotetext{
Received June 8, 2011; revised July 14, 2011; accepted July 19, 2011

Author contributions: G.B.C.,M.S., and J.F.L. designed research;G.B.C. performed research;G.B.C., M.S., and J.F.L. analyzed data; G.B.C. and J.F.L. wrote the paper.

This work was supported by the Gatsby Charitable Foundation (Grant GAT2579/GAT2623), the Wellcome Trust (Grant 084364/Z/07/Z), Deafness Research UK (Grant 412:UEl:JL), and the Biotechnology and Biological Sciences Research Council (Grant BB/H006958/1). We thank Dr. Lucy Anderson for helpful conversations and assistance with histology.

The authors declare no competing financial interests.

This article is freely available online through the J Neurosci Open Choice option.

Correspondence should be addressed to Jennifer F. Linden, UCL Ear Institute, 332 Gray's Inn Road, London WC1X 8EE, UK. E-mail: j.linden@ucl.ac.uk.

DOI:10.1523/JNEUROSCI.2863-11.2011

Copyright $\odot 2011$ the authors $\quad 0270-6474 / 11 / 3112837-12 \$ 15.00 / 0$
}

Mendelson et al., 1997; Sugimoto et al., 1997; Wallace and Palmer, 2008). More recent investigations have reported intriguing evidence for laminar differences in complex response properties, such as coding sparsity (rat: Sakata and Harris, 2009) and spectrotemporal nonlinearity (cat: Atencio et al., 2009). These findings suggest that laminar computations in auditory cortex may involve more abstract sound features than instantaneous frequency, loudness or bandwidth.

Efforts to define those laminar computations have been undermined by the fact that the function of core auditory cortex itself is unclear. In contrast to primary visual cortex, where the uniquely cortical response property of orientation selectivity provides clear evidence for a functional role in form processing, auditory cortex contains neurons with response properties that are very similar to those in earlier stages of the extensive subcortical auditory pathway (King and Nelken, 2009). The most obvious difference between cortical and subcortical response properties is that the range of responses often differ; in particular, cortical neurons show sensitivity to sounds over longer timescales (up to many seconds; Ulanovsky et al., 2004). Based on this observation, Nelken et al. (2003) have argued that the primary role of the auditory cortex is to integrate sound information over long time periods. If so, and assuming interlaminar processing is involved in this computation, then sensitivity to the temporal context in which a sound occurs should vary across the depth of the cortex.

Here we show that temporal response properties of neurons vary with cortical depth in mouse primary auditory cortex (AI) and anterior auditory field (AAF). Recording neural responses 
simultaneously across cortical depth in both AI and AAF, we found systematic depth dependencies in responses to secondand-later noise bursts in slow (1-10 bursts/s) trains of noise bursts, as well as differences between the two cortical areas in responsiveness to the slowest train rates. These results could arise from laminar and area differences in depression at feedforward and recurrent synapses, and are consistent with the hypothesis that temporal processing over slow time scales is an integral function of auditory cortex.

\section{Materials and Methods}

All experiments were performed in accordance with the United Kingdom Animal (Scientific Procedures) Act of 1986. Sixteen male CBA/Ca mice between 8 and 21 weeks of age were used in these experiments. The $\mathrm{CBA} / \mathrm{Ca}$ inbred strain was chosen because it is more resistant to agerelated hearing loss than other common laboratory strains (Zheng et al., 1999). Extracellular electrophysiological recordings were taken from the right cortex of 10 mice; an additional six animals were used to confirm histologically the laminar location of the reversal in polarity of local field potentials, which was used as a cortical depth reference for all recordings.

Acoustic stimulation. Experiments were conducted in an acoustic isolation booth (Industrial Acoustics Company). All stimuli were presented free-field (FF1 speakers, Tucker-Davis Technologies) to the ear contralateral to the recording site, with a sound-attenuating plug inserted into the ipsilateral ear. Before the start of each experiment, the transfer function of the speaker was measured with a microphone (4939, Brüel and Kjær) placed at the location of the animal's ear with the animal in place. This function was used to calibrate individual tones so that the overall output of the speaker was flat across frequency to $\pm 3 \mathrm{~dB}$. Frequency response areas for each recording site were estimated using 100 ms tones ranging from 4 to $90 \mathrm{kHz}$ in frequency, 10 tones per octave, and from 5 to $70 \mathrm{~dB}$ SPL in level in $5 \mathrm{~dB}$ steps.

The noise train stimulus consisted of eight $25 \mathrm{~ms}, 65 \mathrm{~dB}$ SPL "noise bursts" with $5 \mathrm{~ms}$ cosine-squared rise/fall gates. Each noise burst was a dense comb of overlaid tones (with random starting phase) spaced 1/24 octave apart and spanning $2-90 \mathrm{kHz}$ in frequency. Noise bursts were regenerated only between penetrations, so that the structure was frozen across all bursts presented during a single penetration. Train rates were 1 , $2,3,4,5,6.25,7.5$, and 10 bursts/s, and 12 repetitions of each condition were presented in a pseudo-random fashion, with $2 \mathrm{~s}$ of silence between trains. We also collected data using 15 and 20 bursts/s stimulus conditions, and $50 \%$ of the trains included a ninth burst occurring at a cycle and a half following the eighth burst, but those data are not presented here.

All stimuli were synthesized using a digital signal processing unit with a 195,312.5 Hz sampling rate (RX-6), attenuated if necessary (PA-5), and then passed through a stereo amplifier (SA-1) (all Tucker-Davis Technologies). Stimuli were designed and controlled using a combination of Matlab (The MathWorks) and OpenEx (Tucker-Davis Technologies).

Surgical procedures. Animals were anesthetized using a mixture of ketamine $(10 \mathrm{mg} / \mathrm{ml})$ and medetomidine $(0.083 \mathrm{mg} / \mathrm{ml})$. Anesthesia was induced with a dose of $0.01 \mathrm{ml} / \mathrm{g}$ body weight of the ketamine/medetomidine mixture. Dexamethasone (10 $\mathrm{mg} / \mathrm{kg}$ body weight) was administered to reduce brain edema and atropine $(0.05 \mathrm{mg} / \mathrm{kg}$ body weight $)$ to reduce bronchial secretions. Ringer's solution was administered throughout the experiment $(\sim 0.1 \mathrm{ml}$ every $1-2 \mathrm{~h})$ to maintain hydration. Anesthesia levels were monitored using whisker twitch and pedal withdrawal reflexes, and anesthesia was supplemented as needed with 0.00375 $\mathrm{ml} / \mathrm{g}$ body weight of the ketamine/medetomidine mixture. Breathing and temperature were monitored, and a homeothermic blanket system (Harvard Apparatus) was used to maintain body temperature at $37.5 \pm 0.5^{\circ} \mathrm{C}$.

Once anesthetized, mice were placed in a nose clamp to immobilize the head and turned onto their left side so that the right auditory cortex was facing upward. The scalp was transected to expose the skull, and a craniotomy was made over the right temporal lobe by removing the skull bounded by the temporal ridge, the lambdoid suture, and the ventral and rostral squamosal suture. Silicone oil was placed over the craniotomy to keep the exposed cortex moist, and multielectrode penetrations were made through the dura.

Recording procedures. Simultaneous extracellular recordings were collected using a silicon multielectrode with 16 sites (each with $177 \mu \mathrm{m}^{2}$ surface area, $\sim 1-2 \mathrm{M} \Omega$ impedance) spaced $100 \mu \mathrm{m}$ apart along a single shank (a1x16-5mml00-177, NeuroNexus Technologies). The multielectrode was inserted orthogonal to the cortical surface and advanced to $\sim 1200 \mu \mathrm{m}$ below the surface. The multielectrode signals were amplified and digitized (Medusa RA16SD, RX-5, Tucker-Davis Technologies), sampling at $25 \mathrm{kHz}$ with a high-pass filter cutoff at $7.5 \mathrm{~Hz}$, and collected using OpenEx (Tucker-Davis Technologies); local field potentials were subjected to an additional low-pass of $300 \mathrm{~Hz}$.

Local field potentials were recorded in response to a train of four 100 $\mathrm{ms}$ noise bursts at 6.67 bursts/s which was otherwise identical to the basic noise train stimulus described above. For each penetration, we identified the channel at which a reversal in the polarity of the local field potential took place. In separate experiments (see below), we confirmed histologically that this reversal in polarity occurred near the layer I/II border, as has previously been demonstrated in the cat auditory cortex (Kral et al., 2000). We attributed a depth of $100 \mu \mathrm{m}$ to that channel based on previous work (Anderson et al., 2009), and assigned depths to the other channels accordingly. The location of multielectrode penetrations within the fields of auditory cortex was determined on the basis of tonotopy and response properties (Stiebler et al., 1997; Linden et al., 2003).

Multiunit activity was stripped of the local field potential by a highpass filter with cutoff at $600 \mathrm{~Hz}$, and action potentials were classified using a latent variable spike-sorting algorithm (Sahani, 1999). Multiunit clusters as reported here were derived by thresholding only, and ignoring the categorization provided by the spike-sorter; single-unit classifications were accepted only if the spike-sorter reported both false-negative and false-positive rates $<5 \%$.

Lesioning and histological procedures. To confirm the laminar location of the reversal in local field potential polarity used as a depth reference, we conducted separate lesioning experiments, with only one NeuroNexus multielectrode penetration to minimize tissue damage. The multielectrode was inserted through a small hole made with a pin vise drill roughly in the middle of the skull region where the craniotomy would have been made for a recording experiment. The local field potential reversal point was determined as usual, and then an electrolytic lesion was placed at the reversal point using a custom-built lesion maker delivering either 4 or $5 \mu \mathrm{A}$ for $5 \mathrm{~s}$. The multielectrode was then withdrawn, and the animal was overdosed with sodium pentobarbital and perfused transcardially with phosphate buffer. The brain was removed, sectioned, and stained for cytochrome oxidase and Nissl substance using methods described previously (Anderson et al., 2009).

Analysis. Firing rate in response to a noise burst was calculated over a $100 \mathrm{~ms}$ window immediately following the onset of the noise burst. Spontaneous rate was estimated from the $1 \mathrm{~s}$ preceding the onset of each train of noise bursts. Temporal precision of noise burst responses was quantified in terms of the SD of spike times in the $100 \mathrm{~ms}$ response window, either for the second-and-later bursts in a train ("within-train spike-time SD") or for the first bursts alone ("first-burst spike-time SD"). For a supplemental analysis, vector strength was computed as described by Goldberg and Brown (1969), and was analyzed only for 10 bursts/s noise burst trains, in which the fixed $100 \mathrm{~ms}$ analysis window used for other analyses was equal to the inter-onset interval between noise bursts.

We quantified the neural response within a train using the "following index" (FI), defined as the mean firing rate in response to the second-andlater bursts in a train of a given rate, divided by the mean firing rate over all the first noise bursts in all trains. This calculation yielded a value of FI for each train rate $r$. The use of a fixed $100 \mathrm{~ms}$ analysis window to compute firing rate in response to each noise burst ensured that the same amount of data was used in FI calculations for all train rates, justifying a direct comparison of FI across train rates. We could therefore analyze, for each cluster recording as well as for population data, the characteristics of the FI-rate function obtained by plotting following indices $\mathrm{FI}(r)$ versus train rates $r$.

The "rolloff corner point", $r_{\text {ro }}$, of the FI-rate function was defined to be the fastest rate such that FI $(r)$ was not significantly lower than 
$\mathrm{FI}\left(1 \mathrm{burst} / \mathrm{s}\right.$ ) for all $r \leq r_{\text {ro }}$ (Kruskal-Wallis test, $\alpha=0.05$ ). If FI $(r)$ values for a given cluster recording were not significantly lower than FI(1 burst/s) for any train rate $r>1$ burst/s, then the recording was excluded from rolloff corner point analysis. Conversely, if FI ( 2 burst/s) was already significantly lower than FI(1 burst/s), then we concluded that the rolloff corner point lay below our range of sampled train rates, and we included the data under "N/A" in Figure 4 (see below). For the purposes of comparing the AI and AAF populations, these N/A points were not rejected, but assigned a value of $r_{\text {ro }}=1 \mathrm{burst} / \mathrm{s}$; results were similar when these points were instead excluded from the analysis.

Similarly, the "floor corner point" $r_{\mathrm{fl}}$ of the FI-rate function was the slowest rate for which $\mathrm{FI}(r)$ was not significantly different from FI(10 bursts/s) for all $r \geq r_{\mathrm{fl}}$. Recordings were excluded from analysis if $\mathrm{FI}(r)$ values were not significantly different from $\mathrm{FI}(10$ burst/s) for any train rate $r<10$ burst/s. However, if FI(7.5 burst/s) was already significantly different from $\mathrm{FI}(10$ burst/s) -i.e., if the floor corner point appeared to lie above our range of sampled train rates-we included the data under N/A in Figure 4 (see below). For comparison of AI and AAF populations, these N/A points were assigned a value of $r_{\mathrm{f} 1}=10 \mathrm{burst} / \mathrm{s}$; results were similar when these points were instead excluded.

To quantify the stability of rolloff or floor corner points within a penetration, we calculated the SD of the corner points for all clusters within that penetration. We then computed the mean of these withinpenetration SDs across all penetrations, and compared this value to a resampled reference distribution generated by recalculating the mean within-penetration SD 10,000 times following repeated randomizations of the assignments of clusters to penetrations. We then estimated the probability of the observed mean within-penetration SD under the null hypothesis of no difference between within-penetration and acrosspenetration variability, based on the number of points in the reference distribution that were equal to or less than the observed data value.

A cluster was declared to have a significantly non-monotonic FI-rate function if for at least one train rate, the FI for that train rate was significantly greater than the FI for the immediately slower train rate $(p<0.01$ with Holm-Bonferroni correction for multiple tests). For every train rate in every cluster, we generated a resampled reference distribution for the difference in FI between that train rate and the immediately slower rate, using 10,000 random permutations of the assignment of second-andlater noise burst responses to either the faster or slower train rate condition. This resampled reference distribution was then used for the significance test, to determine the probability of the observed difference in FI values between the two adjacent train rates under the null hypothesis of no difference in neural responses at the two rates.

A cluster was said to show an "augmenting response" if for at least one train rate, the mean firing rate for second-and-later bursts was significantly greater than the mean firing rate over all first bursts $(p<0.01$ with Holm-Bonferroni correction for multiple tests). For every train rate in every cluster, the significance of the difference between responses to second-and-later bursts and responses to all first bursts was determined relative to a resampled reference distribution obtained by randomizing assignments of responses to the second-and-later-burst versus first-burst conditions 10,000 times.

All statistical tests were two-tailed unless noted otherwise. For comparison of percentages of clusters between cortical areas, we used Fisher's exact test. Depth dependencies in the incidence of FI-rate function nonmonotonicity or augmenting responses were assessed using Pearson's correlation test, because of the relatively small number of observations involved. In all other analyses, depth dependencies were evaluated using the nonparametric Kruskal-Wallis rank-sum test, which tests the null hypothesis that all the groups have the same distribution. Rejection of the null hypothesis justifies the use of pairwise statistical tests; however, because of the large number of such comparisons in our dataset and because comparisons between particular depths are of less interest here than overall depth-dependent trends, we instead report the general trend as measured by the $95 \%$ confidence interval of the slope of a linear regression on the data (slope CI). Pairwise comparisons were performed to verify that significant differences between different depths were consistent with regression trends, but those statistics are not reported here.
To ensure that depth dependencies within simultaneous recordings from individual penetrations were not obscured by variability across sequential recordings and different penetrations, we also tested "penetration-relative" data: for all clusters recorded simultaneously in the same penetration, we subtracted the value of the observation at a depth of $400 \mu \mathrm{m}$ (likely corresponding to layer IV) from the observations at all other depths in that penetration. We then conducted a KruskalWallis test on these penetration-relative values, excluding the $400 \mu \mathrm{m}$ condition. This procedure required discarding data from 3 penetrations in $\mathrm{AI}$ and 1 penetration in AAF for which there was no cluster recorded at $400 \mu \mathrm{m}$. Results are given in the text for statistical tests on both absolute and penetration-relative data values where appropriate.

\section{Results}

For all recordings, we used reversal in the polarity of local field potentials (LFPs) as a depth reference. In six animals used to confirm the cortical depth of this reference, electrolytic lesions placed at the LFP reversal point were histologically localized within $150 \mu \mathrm{m}$ of the cortical surface, near the layer I/II border (example: Fig. $1 A-C$ ). We therefore attributed a depth of $100 \mu \mathrm{m}$ to the channel at the LFP reversal point, and assigned depths to the other channels in the multielectrode accordingly. Depth measurements should be interpreted as $\pm 50 \mu \mathrm{m}$ given estimated uncertainty in the localization of the LFP reversal point and the 100 $\mu \mathrm{m}$ spacing of electrode sites on the multielectrode probe. Based on previous analyses of laminar structure in the mouse auditory cortex (Anderson et al., 2009), depths of up to $300 \mu \mathrm{m}$ likely correspond to supragranular layers (I, II and III), 300-500 $\mu \mathrm{m}$ to granular layers (lower layer III and layer IV), and $500 \mu \mathrm{m}$ and deeper to infragranular layers (V and VI).

Recordings of neural responses to $1-10$ bursts/s trains of noise bursts were collected across the depth of the primary auditory cortex and anterior auditory field in 10 male CBA/Ca mice. Due to the time constraints imposed by the long stimulus train protocol, we were not able to present enough repetitions of tonal stimuli to support an in-depth analysis of frequency-intensity response area parameters, but only enough to determine whether tonotopy and basic response properties indicated that the penetration was in AI or AAF. A total of 432 multiunit clusters were recorded in 60 penetrations in $\mathrm{AI}$ and 438 multiunit clusters in 58 penetrations in AAF. In all but two animals recordings were obtained in both $\mathrm{AI}$ and $\mathrm{AAF}$, in a minimum of 8 penetrations and a maximum of 20 per animal. From the two animals for which only AAF recordings were collected, we obtained 2 and 5 penetrations respectively.

Clusters were recorded at all depths in most penetrations, with a small bias toward intermediate depths (Fig. 1D,E). Spikesorting yielded 87 single units in AI and 83 single units in AAF. The single units were not evenly sampled across depth; their distribution was strongly biased toward deeper depths. Therefore, for analysis of depth-dependent effects we focus on the thresholded multiunit cluster data. Results of response latency analysis at different depths were inconclusive and are not presented here, but were generally consistent with previous observations that neurons in infragranular layers have the shortest response latencies in rodents (Sugimoto et al., 1997; Shen et al., 1999; Sakata and Harris, 2009).

\section{Train-dependent response properties}

Neural responses to trains of repeated stimuli are sometimes quantified in terms of firing rates calculated over the entire stimulus train; however, this measure conflates train rate with firing rate evoked by each stimulus within the train. Since we were primarily interested in how neural responses to noise bursts 


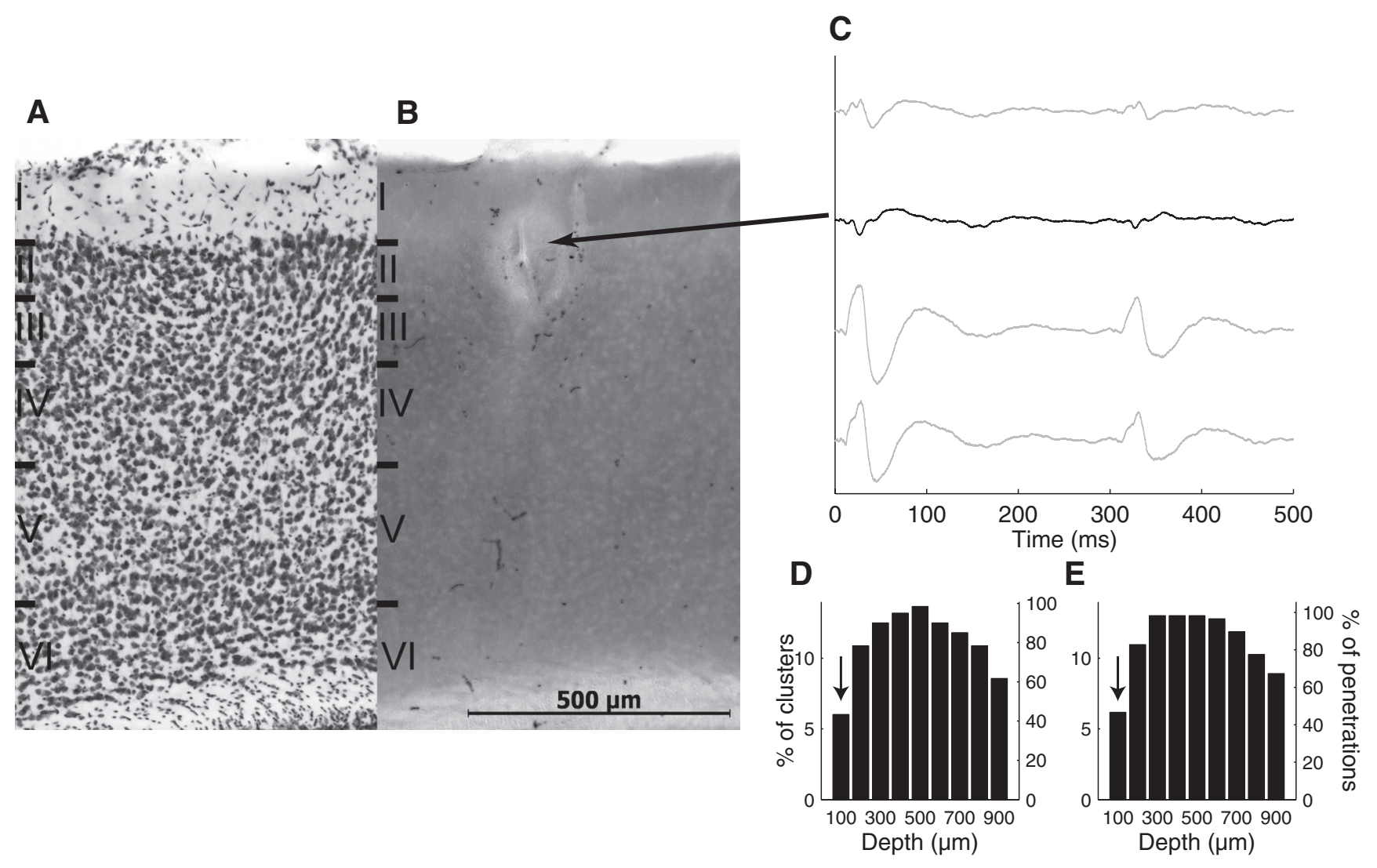

Figure 1. Histological confirmation of depth of LFP reversal point. $\boldsymbol{A}$, A section stained for Nissl substance, used to identify laminar boundaries. $\boldsymbol{B}$, The adjacent section in the series from this animal, stained for cytochrome oxidase, reveals a lesion near the layer I/II boundary (indicated by the arrowhead). C, LFP traces demonstrating the reversal point (black) where the lesion was placed. Channels both above and below the reversal (gray) are shown for illustration. $\boldsymbol{D}, \boldsymbol{E}$, Also plotted is the distribution of multiunit clusters by depth for both Al (D) and AAF (E), in percentage of clusters (left axis) and percentage of penetrations which included a cluster at that depth (right axis; note that only one cluster can exist per depth per penetration, so the two axes are linearly related).

within the context of a train compared with neural responses to isolated (first) noise bursts, we instead quantified responses using a following index (FI) (see Materials and Methods) defined as the mean firing rate evoked by second-and-later noise bursts within a train, relative to the mean firing rate evoked by all first bursts. Firing rate in response to a noise burst was always measured over a fixed $100 \mathrm{~ms}$ interval following noise burst onset. For all train rates, this $100 \mathrm{~ms}$ response interval was less than or equal to the onset-to-onset interval for noise burst presentations. Therefore, we could compare following indices across train rates without confounding train rate with firing rate evoked by individual noise bursts within a train. We plotted following index versus train rate functions (FI-rate functions) for recordings at each cortical depth to examine how train-dependent response properties varied across cortical depth. Example responses to noise burst trains at three representative train rates, for clusters at three different cortical depths, are shown as rasters in Figure $2 A-C$ and as poststimulus time histograms (PSTHs) in Figure $2 D-F$; full FI-rate functions for these three cluster recordings are displayed in Figure $2 G$.

We also attempted to compare responses to second, third, etc. noise bursts within trains to determine the time course of adaptation or facilitation. We found no evidence for significant differences between responses to second-and-later noise bursts; however, the number of data points for each of these noise bursts within a train was comparatively low, and results were noisy and therefore somewhat inconclusive. As illustrated in Figure 2, however, individual example recordings also revealed little evidence for differences between responses to second-and-later noise bursts within a train, whereas responses to noise bursts within a train were often distinctly different from responses to first bursts.

\section{Following index magnitude}

Following index magnitude varied systematically across cortical depth in both $\mathrm{AI}$ and AAF, as demonstrated in the FI-rate functions for two representative AI and AAF penetrations in Figure $3 \mathrm{~A}$, and in $\mathrm{AI}$ and $\mathrm{AAF}$ population average FI-rate functions in Figure $3 B$. In both AI and AAF, following index magnitude was not only greater for slow (1-5 bursts/s) than for fast (6.25-10 bursts/s) train rates, but also greater at mid-to-superficial depths $(<500 \mu \mathrm{m})$ than at deeper depths $(>500 \mu \mathrm{m})$. Indeed, peak FI in cluster recordings was significantly depth-dependent, decreasing with increasing depth in both AI and AAF (Fig. 3C, both areas $p<$ $10^{-6}$, Kruskal-Wallis test, penetration-relative (see Materials and Methods) $p<10^{-6}$; AI slope CI $[-0.4,-0.2]$ per mm, AAF slope CI $[-0.4,-0.3]$ per $\mathrm{mm}$ ).

There was an apparent reversal in the relationship between FI magnitude and cortical depth at the fastest train rates, with larger FI values at deeper depths (Fig. 3A,B). However, neural responses generally did not lock to within-train noise burst presentations at higher train rates; for example, there was no significant depth dependence in vector strength (see Materials and Methods) of neural responses to 10 bursts/s noise burst trains. Therefore, the depth dependence in following index magnitude at higher rates appeared to arise from differences in sustained activity during fast noise burst trains, rather than from differences in the degree of locking to individual noise burst presentations in fast trains. 
A
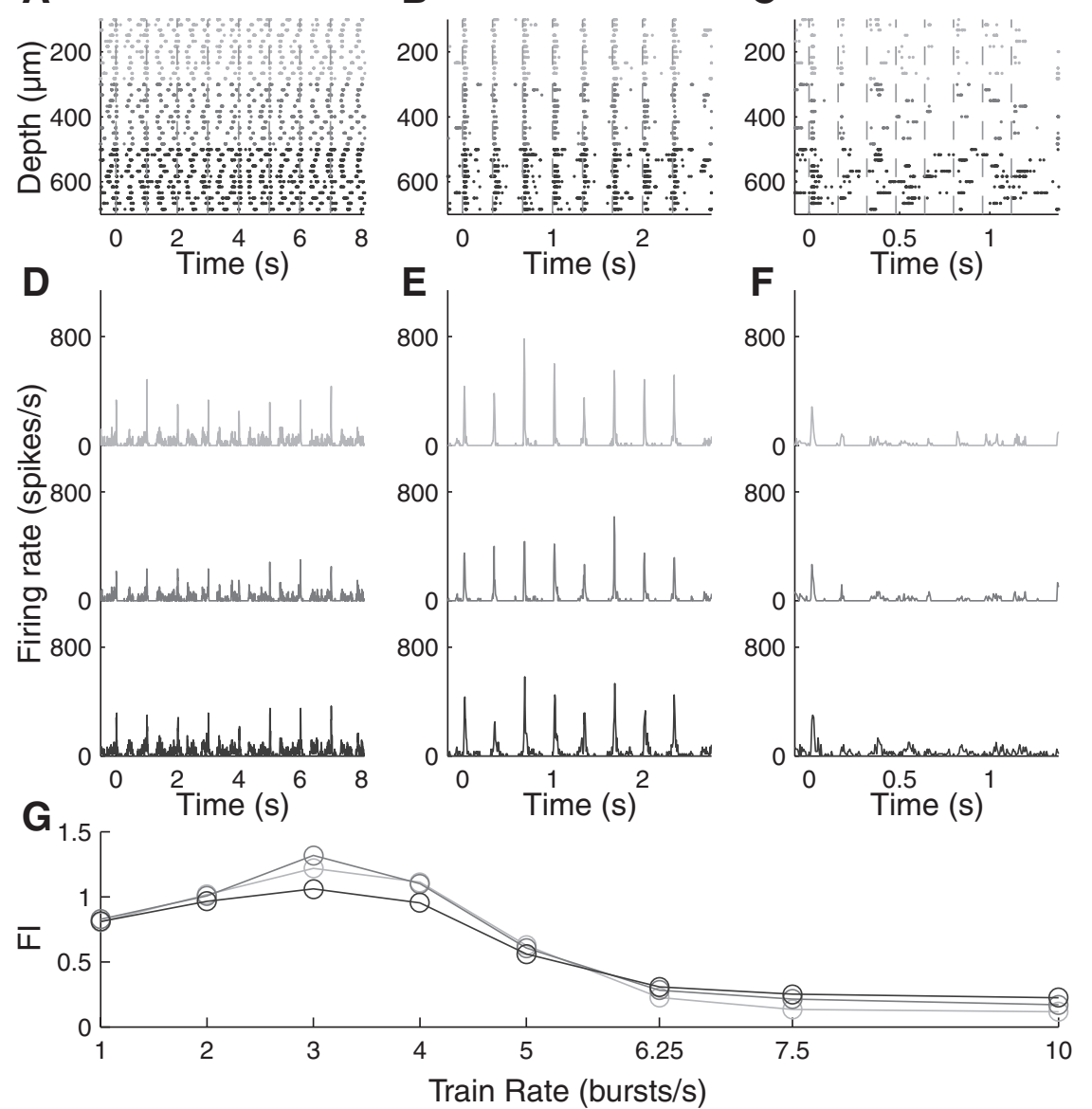

Figure 2. Example data from one multielectrode penetration. $\boldsymbol{A}-\boldsymbol{C}$, Spike rasters show simultaneous multiunit cluster recordings from 200,400, and $600 \mu \mathrm{m}$ depths in the same AAF penetration, for representative noise burst train rates of $1(\boldsymbol{A}), 3(\boldsymbol{B})$, and 6.25 (C) bursts/s. Rasters have been collapsed into $5 \mathrm{~ms}$ bins and plotted as peristimulus time histograms in $\boldsymbol{D}-\boldsymbol{F}$, using the same gray-scale scheme as in $\boldsymbol{A}-\boldsymbol{C}$ to indicate recording depth. In $\mathbf{G}$, the following index $\mathrm{Fl}$ is shown for the same three recording depths (gray-scale scheme as in $\boldsymbol{A}-\boldsymbol{F}$ ), and plotted for the full range of stimulus train rates.

\section{Rolloff and floor corner points}

As the population averages suggest (Fig. $3 B$ ), FI-rate functions generally rolled off between 3 and 6.25 bursts/s, and stabilized at higher train rates. To characterize the shapes of these functions, we borrowed terms used to describe low-pass filters, defining the rolloff corner point $r_{\text {ro }}$ as the fastest train rate before the rolloff in FI values, and the floor corner point $r_{\mathrm{fl}}$ as the slowest train rate after that rolloff (Fig. $4 A$; see Materials and Methods for details).

In both $\mathrm{AI}$ and $\mathrm{AAF}$, rolloff corner points $r_{\text {ro }}$ were significantly depth dependent (Fig. 4B; $p<0.005$, Kruskal-Wallis test, penetration-relative $\left.p<10^{-4}\right)$. Rolloff began at faster train rates in more superficial layers, and slower train rates in deeper layers (both areas, slope CI $[-2,-0.8]$ bursts/s per mm). In contrast, floor corner points $r_{\mathrm{fl}}$ did not vary significantly with cortical depth when data were pooled across penetrations $(p>0.05$ for AI, $p>0.01$ for AAF, Kruskal-Wallis test), and showed little depth dependency even when evaluated within each penetration relative to the value at $400 \mu \mathrm{m}$ (penetration-relative n.s. for AAF, $p<10^{-5}$ for AI but with $r_{\mathrm{fl}}$ occurring at slower rates compared with the $400 \mu \mathrm{m}$ depth at only the most superficial and the deepest depths). Thus while rolloff corner points showed systematic variation with cortical depth, floor corner points were comparatively consistent across depth.
Despite this difference in depth dependence, both $r_{\text {ro }}$ and $r_{\mathrm{fl}}$ were more similar for clusters within the same penetration than across penetrations $\left(p<10^{-4}\right.$ for both corner points and both areas, see Materials and Methods). Neural responses therefore showed similar sensitivity to slow train rates within an orthogonal penetration through cortex, but the slope of the FI-rate function became shallower with increasing depth, as the rolloff corner point shifted to slower train rates.

In addition to these differences between superficial and deep layers, we also observed differences between the two cortical areas. Rolloff began at slower train rates in AI than in AAF; the $r_{\text {ro }}$ distributions for AI and AAF were significantly different (Fig. 4C; $p<10^{-6}$, KruskalWallis test). Moreover, clusters for which $r_{\text {ro }}$ was 1 burst/s or slower (indicating that rolloff occurred at a train rate below the slowest rate tested) were more common in AI than in AAF (AI: 100 of 421, 24\%; AAF: 66 of 413, 16\%; Fisher's exact test, $p<$ $0.01)$. Floor corner points also occurred at slower train rates in AI than in AAF (Fig. $4 D ; p<10^{-6}$, Kruskal-Wallis test). The majority of AI clusters had $r_{\mathrm{fl}}$ at train rates of 6.25 bursts/s or slower, while in AAF, $r_{\mathrm{fl}}$ tended to occur at train rates of 6.25 bursts/s or faster. There were very few clusters in either area for which $r_{\mathrm{fl}}$ did not occur within the sampled range of train rates (AI: 12 of 417, 3\%; AAF: 23 of 418, $6 \%$; Fisher's exact test, n.s.).

\section{Non-monotonicity with train rate}

Previous studies in the rat (Kilgard and Merzenich, 1999) have demonstrated that some auditory cortical neurons have non-monotonic FI-rate functions. In other words, for some neurons, responses to second-and-later tones in a train did not decrease monotonically with increasing train rate, but instead were stronger at moderately fast train rates than at slower (or much faster) train rates. To identify clusters with nonmonotonic FI-rate functions in mouse auditory cortex, we performed a statistical test (see Materials and Methods) to determine whether the FI for at least one train rate was significantly greater than the FI for the immediately slower rate (Fig. 5A). In both AI and AAF, we found more clusters with non-monotonic FI-rate functions than would be expected by chance at our significance threshold of 0.01 (AI: 22 of 432 clusters over 9 of 60 penetrations; AAF: 34 of 438 clusters over 10 of 58 penetrations). Moreover, in both areas, the train rate at which the non-monotonicity occurred was almost exclusively 3 bursts/s (Fig. $5 B$ ). In AI, there was no clear depth dependence in the distribution of clusters with non-monotonic FI-rate functions (Fig. 5C, left), while in AAF, these clusters seemed to occur most often at $\sim 400 \mu \mathrm{m}$ (Fig. $5 C$, right). However, in both areas, there was a significant depth dependence in the magnitude of the non-monotonicity; the difference in FI between the faster and immediately slower train rate was greatest at more superficial depths (Fig. 5D; $p<0.01$, Pearson's correlation test). 

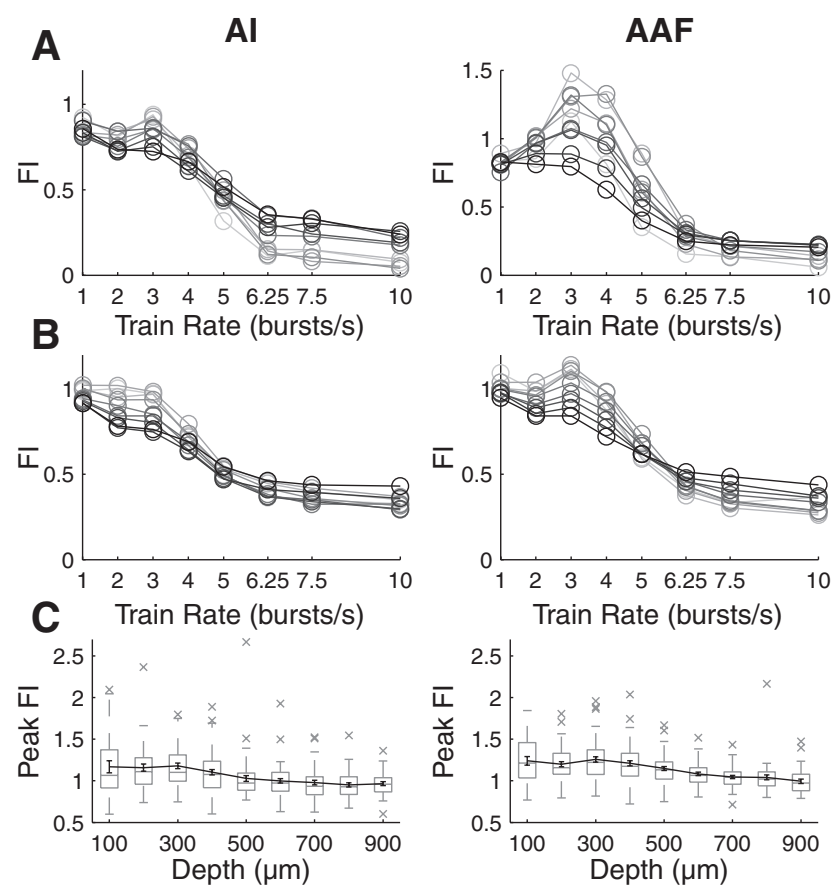

Figure 3. Following index magnitude varies with cortical depth. $\boldsymbol{A}$, Data from two representative penetrations from Al (left) and AAF (right), illustrating Fl-rate functions. Each curve represents a different recording depth, with lighter curves indicating more superficial depths. The AAF example is the same as in Figure 2, with more depths included. $\boldsymbol{B}$, Population averages of Fl (mean rate of response to second-and-later bursts normalized by mean rate of response to first bursts) as a function of train rate for AI (left) and AAF (right). Error bars are omitted for visual clarity. C, The peak following decreases with depth in both Al and AAF (Kruskal-Wallis test, $p<$ $10^{-6}$; black lines give mean \pm SEM; gray lines in box give the median; box extends from the 25 th to 75 th percentile; whiskers show the extent of the data out to at most half again the interquantile distance; outliers are marked with $\mathrm{x}$ ).

\section{Augmenting responses}

For some neurons, non-monotonicity in the FI-rate function appeared to arise from augmenting responses at moderate train rates; stronger firing rates were elicited by noise bursts within the context of a train than by the bursts that initiated the train. These augmenting responses represent a particularly striking example of a train-dependent response property. Clusters with augmenting responses were defined as those for which the response to second-and-later bursts was significantly greater than the response to all first bursts (i.e., FI $>1$ ) for at least one train rate (see Materials and Methods). In AAF, augmenting responses were observed in 24 of 438 clusters ( 11 of 60 penetrations), predominantly at train rates of 3 bursts/s (Fig. 6A); 16 also met the condition for non-monotonicity as defined above and in Figure 5. The augmenting responses occurred mainly in mid-to-superficial layers, primarily $\sim 300-400 \mu \mathrm{m}$ below the cortical surface (Fig. $6 B$; distribution across depth significantly different from distribution of all clusters across depth, $p<10^{-4}, \chi^{2}$ goodnessof-fit test). However, the magnitude of augmenting decreased with increasing depth (Fig. 6C; $p<0.05$, Pearson's correlation test), so augmenting was most pronounced in the more superficial layers.

Examples of PSTHs for AAF clusters with augmenting responses are shown in Figure 7, A and B. In particular, Figure 7A demonstrates that augmenting responses may be evident from as early as the second burst in a stimulus train. Augmenting responses were observed not only in multiunit clusters but also in single-unit recordings. Of the 87 single units identified in AAF

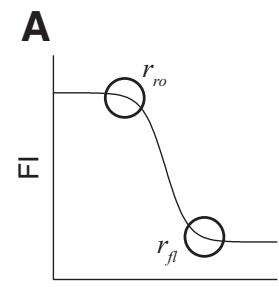

Train Rate (bursts/s)

Al

B
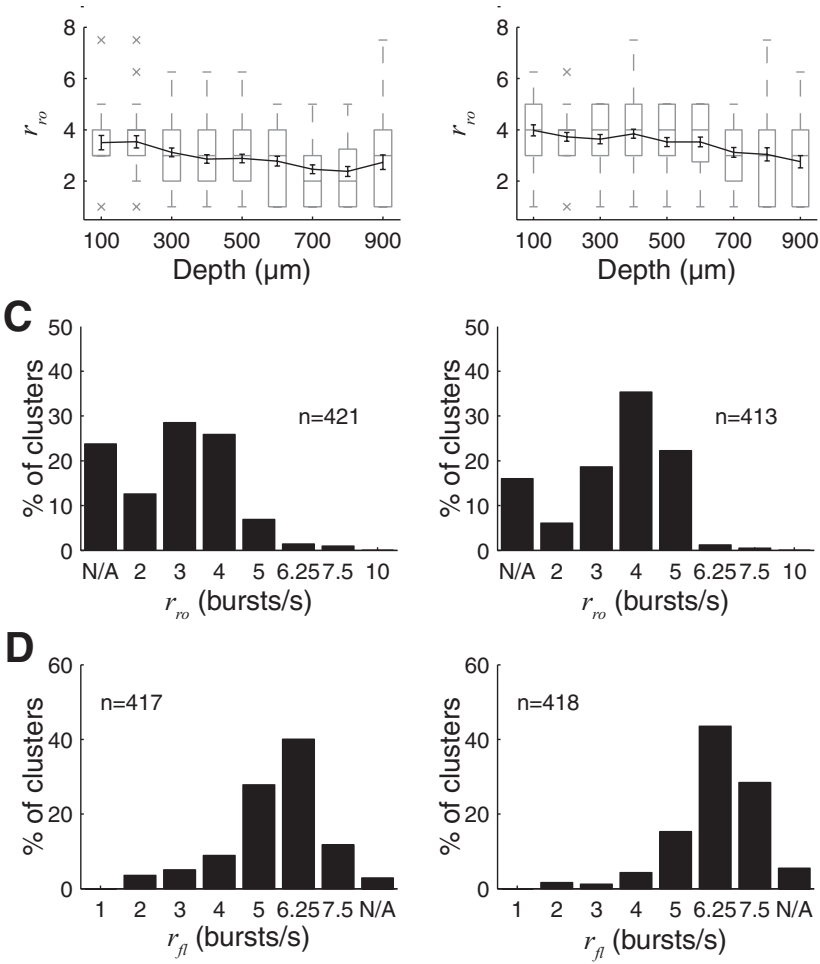

Figure 4. Corner points for FI-rate functions in Al and AAF. A, Schematic illustration of rolloff $\left(r_{\text {ro }}\right)$ and floor $\left(r_{f f}\right)$ corner points for an idealized Fl-rate function. $\boldsymbol{B}, r_{\text {ro }}$ was depth dependent in both Al and AAF (Kruskal-Wallis test, $p<0.005$ for both); the rolloff in Fl-rate functions began at slower rates in deeper clusters. Black lines give mean \pm SEM; gray lines in box give the median; box extends from the 25th to 75th percentile; whiskers show the extent of the data out to at most half again the interquantile distance; outliers are marked with x. C, D, Area differences in $r_{r 0}$ and $r_{f l}$ distributions. Rolloff ( $\boldsymbol{C}$ began at slower rates in Al (left; 421 clusters) than in AAF (right; 413 clusters). Similarly, FI reached its floor value $(\boldsymbol{D})$ at slower rates in AI ( 417 clusters with defined $r_{\mathrm{fl}}$ ) compared with AAF (418 clusters). However, this effect was not depth dependent (Kruskal-Wallis test, n.S.; data not shown). N/A, Corner point did not occur within the range of train rates presented in this study.

recordings through spike sorting, 5 showed augmenting responses, a proportion similar to that obtained in multiunit cluster recordings. Like augmenting responses in multiunit clusters, these single-unit augmenting responses occurred primarily at train rates of 3 bursts/s ( 5 of 5 units) and at cortical depths of $300-400 \mu \mathrm{m}$ ( 3 of 5 units; data not shown).

Augmenting responses in AAF appeared to be associated with a release of prolonged suppression of neural activity following the brief stimulus-evoked increase in firing rate. Figure $7 C$ shows PSTHs averaged over all responses to noise bursts separated by at least $500 \mathrm{~ms}$ (i.e., noise burst responses from 1 and 2 bursts/s stimulus conditions), normalized such that spontaneous activity is zero and the maximum is one, for AAF clusters with and without significant augmenting responses. This figure reveals that 
A

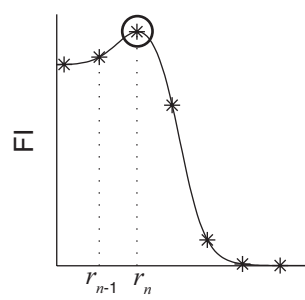

Al

Train Rate (bursts/s)

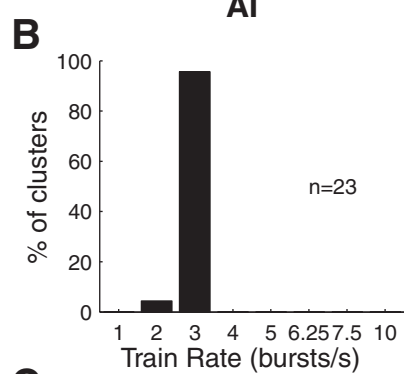

C

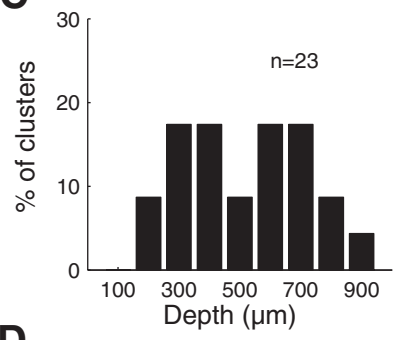

D

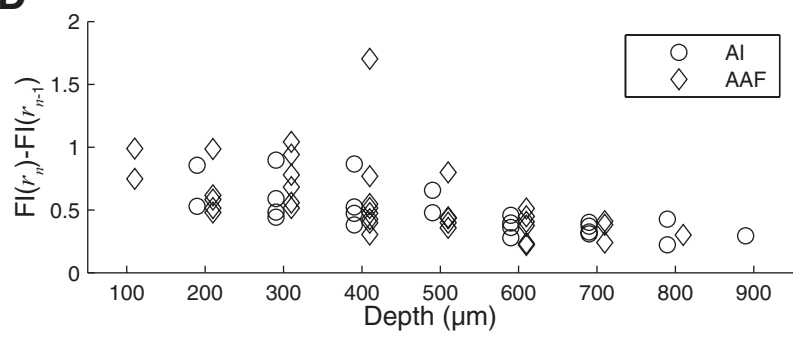

Figure 5. Non-monotonicity in Fl-rate functions. $\boldsymbol{A}$, Idealized non-monotonic Fl-rate function; non-monotonicity is circled and identified as $r_{n}$. $\boldsymbol{B}$, In both Al (left) and AAF (right), non-monotonic Fl-rate functions occurred almost exclusively at train rates of 3 bursts/s. C, The distribution of clusters with significantly non-monotonic Fl-rate functions was depth dependent in AAF (with a peak near $400 \mu \mathrm{m}$ ) but not in Al. D, However, in both Al (circles) and AAF (diamonds), the magnitude of nonmonotonicity was depth dependent (Pearson's correlation test, $p<0.01$ ), with the largest nonmonotonicincrease in Fl between adjacent train rates occurring at more superficial depths. Magnitude of non-monotonicity (on $y$-axis) is defined as the difference in $\mathrm{Fl}$ between the rate identified as nonmonotonic and the immediately slower rate (i.e., $\left.\mathrm{F}\left(r_{\mathrm{n}}\right)-\mathrm{FI}\left(r_{\mathrm{n}-1}\right)\right)$. compared with the activity of clusters without augmenting responses, the activity of clusters with augmenting responses was more deeply suppressed following the initial excitatory response to a noise burst, and rebounded to a higher level of activity upon release of the suppression. Indeed, for clusters with augmenting responses, this "rebound" activity exceeded the spontaneous firing rate. Moreover, the peak of the rebound occurred 300-350 $\mathrm{ms}$ after stimulus onset, a time interval that matches the period of the 3 bursts/s stimulus trains for which augmenting responses (and non-monotonicity in FI-rate functions) were most pronounced.

In AI, 6 of 432 clusters in 6 distinct penetrations showed augmenting responses; given our significance threshold of 0.01 , this proportion is consistent with a number obtainable by chance. We therefore cannot conclude that augmenting responses were present in AI as well as AAF. However, the putative augmenting responses in AI occurred at or near 3 bursts/s, and primarily at a depth of $300 \mu \mathrm{m}$ as did the augmenting responses in AAF. Moreover, each of the AI clusters with augmenting responses occurred in a different penetration ( 6 of 60 penetrations) spread over 5 of 10 of the mice used in our study. Thus while our study lacked the statistical power to demonstrate the presence of augmenting responses in AI, it is possible that such responses are not limited to AAF.

\section{Other response properties}

Post-activation suppression

One of the striking features in the PSTHs of Figure $7 \mathrm{C}$ is the prolonged suppression of activity $\sim 100-250 \mathrm{~ms}$ after stimulus onset, following the brief stimulus-evoked increase in firing rate. As explained above, augmenting responses seemed to be associated with particularly deep post-activation suppression, and subsequent rebound activity $\sim 300-350 \mathrm{~ms}$ after stimulus onset. We wondered whether the magnitude of post-activation suppression, like the magnitude of augmenting responses (Fig. $6 \mathrm{C}$ ), might be greater in more superficial than in deeper layers. To analyze depth-dependence of post-activation suppression, we grouped recordings into coarse depth categories and computed average normalized PSTHs for all responses to noise bursts separated by at least $500 \mathrm{~ms}$ (as in Fig. 7). We found that post-activation suppression was stronger in mid-to-superficial than deep layers (Fig. $8 \mathrm{~A}$ ). In both $\mathrm{AI}$ and $\mathrm{AAF}$, the mean value of the normalized PSTH over the 100-250 ms period after stimulus onset was significantly depth dependent, increasing with increasing depth (Fig. 8 B; AI $p<$ $10^{-5}$, AAF $p<0.01$, Kruskal-Wallis test; AI slope CI [0.03, 0.08] per $\mathrm{mm}$, AAF slope CI $[0.008,0.05]$ per $\mathrm{mm})$. This analysis of extracellular recording data most likely underestimates the true depth dependence of post-activation suppression, since the maximum suppression observable in extracellular recordings is limited by spontaneous firing rates, which shows a different depth dependence from that we observe for post-activation suppression (see below).

\section{Precision of spike timing}

The precision of spike timing in responses to noise bursts varied with cortical depth in both AI and AAF. We quantified spike timing precision by measuring the SD of spike times in the $100 \mathrm{~ms}$ analysis windows after noise burst onsets; this measure is inversely related to timing precision. Spike-
Figure 6. Augmenting responses in AAF.A,Augmenting responses to second-and-laterbursts in a noise burst train occurred primarily at slower train rates near 3-4bursts/s. B, Augmenting responses were also restricted primarily to mid-to-superficial layers, particularly $300-400 \mu \mathrm{m}$ below the cortical surface (distribution significantly differentfrom distribution of all dusters across depth, $\chi^{2}$ goodness-of-fit, $p<10^{-4}$ ). $C$, Themagnitude of the augmenting response was negatively correlated with depth (Pearson's correlation test, $p<0.05$ ). 
A

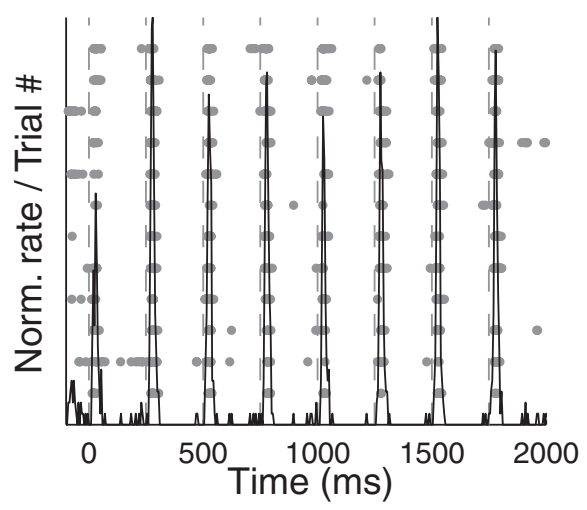

B

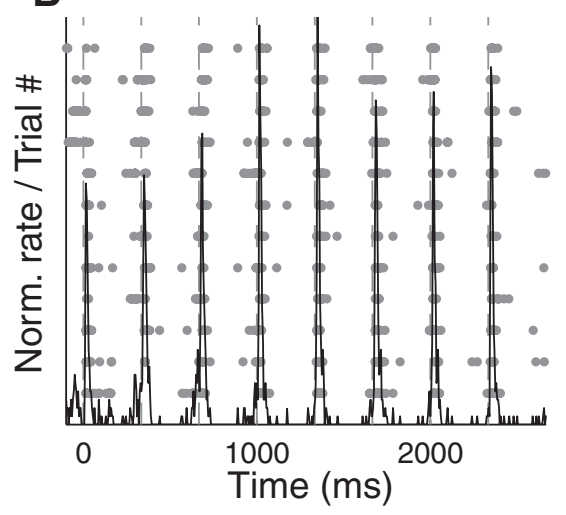

C

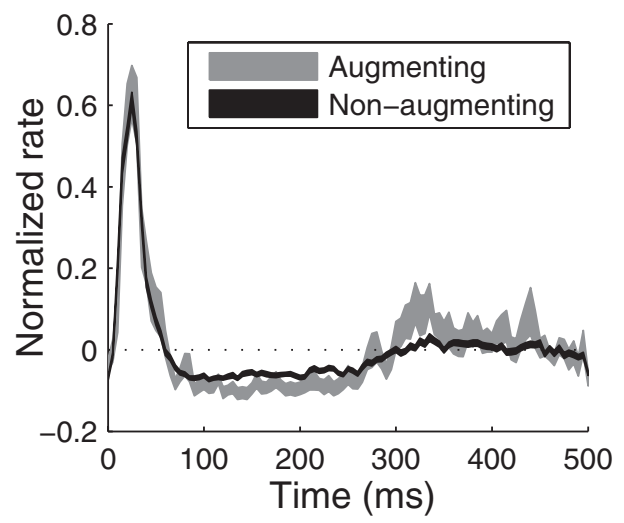

Figure 7. Time course of augmenting responses. $A, B$, Example augmenting responses at 4 bursts/s ( $\boldsymbol{A}$; cluster m00167p32d300) and 3 bursts/s ( $\boldsymbol{B}$; cluster m00203p2d400). PSTHs (black) are superimposed over spike rasters (gray dots). $\boldsymbol{C}$, Average normalized PSTH for all AAF clusters with (gray) or without (black) significant augmenting responses. Each cluster PSTH contributing to these averages was computed for responses to all noise bursts separated by at least $500 \mathrm{~ms}$, and normalized by subtracting spontaneous rate and scaling the maximum value to one. Width of traces gives mean \pm SEM; dotted line at zero represents spontaneous activity level. The clusters with augmenting responses show more post-activation suppression, and greater rebound activation $\sim 300-350 \mathrm{~ms}$ after stimulus onset.

time SD was computed from cluster recordings at different cortical depths, either for responses to noise bursts within trains (Fig. $9 A$ ), or for responses to "first bursts" initiating trains (see Materials and Methods for details). In both AI and AAF, the minimum within-train spike-time SD differed significantly between clusters recorded at different depths (Fig. 9B; Kruskal-Wallis test results, AI: $p<10^{-5}$, penetration-relative $p<10^{-9}$; AAF: $p<10^{-10}$, penetration-relative $p<10^{-10}$; similar results obtained for fixed
$1-5$ bursts/s train rates rather than for the minimum across train rates). More specifically, in both AI and AAF, minimum withintrain spike-time SD increased with increasing cortical depth (AI slope CI $[4,8] \mathrm{ms} / \mathrm{mm}$, AAF slope CI $[6,10] \mathrm{ms} / \mathrm{mm})$; that is, spike timing precision decreased with depth. Spike-time SD for first-burst responses showed similar depth dependence (data not shown). Indeed, the normalized difference in spike-time SD between first-burst responses and within-train responses was near zero and showed no significant depth dependence (Fig. 9C, $p>$ 0.1 using minimum within-train spike-time SD across trains; similar results of $p>0.2$ obtained using within-train spike-time $\mathrm{SD}$ for fixed 1-5 bursts/s train rates). Thus there was no evidence for enhancement of either spike timing precision or depth dependence of timing precision by entrainment of neural responses to noise bursts within a stimulus train.

Although spike timing precision showed similar dependence on cortical depth in both AI and AAF, precision was higher overall in AAF than in AI, at least at slow stimulation rates (Fig. 9A). First-burst spike-time SD and minimum within-train spike-time SD were significantly lower in AAF than in AI at all cortical depths $\left(p<10^{-4}\right)$. At the highest train rates, within-train spike-time SDs in both AI and AAF converged toward the $28.8 \mathrm{~ms}$ value expected if spike times were uniformly distributed over the 100 ms analysis windows (Fig. 9A, dotted line). This observation, which held for clusters in both AI and AAF and at all cortical depths, corroborates our earlier finding that cluster responses at faster train rates showed very little locking to individual noise bursts within the trains (see Following index magnitude, above).

\section{Spontaneous rates and first-burst responses}

In both $\mathrm{AI}$ and $\mathrm{AAF}$, spontaneous firing rates were dependent on cortical depth, as were stimulus-driven firing rates elicited by the first noise burst in a train (Fig. $10 A, p<10^{-6}$, Kruskal-Wallis test, penetration-relative $\left.p<10^{-6}\right)$. The general observed trend was for spontaneous rates and first-burst responses to increase with depth to a peak that fell $\sim 600 \mu$ m below the cortical surface (likely layer V), and then to decrease at greater depths, as has been reported in other studies (Sakata and Harris, 2009). In contrast, the previously described train-dependent response properties of peak FI, rolloff corner point, magnitude of non-monotonicity in the FI-rate function, and augmenting response magnitude all decreased systematically with increasing cortical depth (as did the essentially train-independent property of spike timing precision). Therefore, depth dependence in spontaneous and driven firing rates could not account for the observed depth dependencies in these other neural response properties. On the other hand, higher firing rates in middle layers may have contributed to the increased likelihood of observing non-monotonic FI-rate functions and augmenting responses in middle layers, since estimation of FI values is more reliable at higher firing rates, and therefore both non-monotonicity in FI-rate functions and augmenting responses might be easier to detect in recordings from middle layers.

Spontaneous rates and first-burst response rates were correlated in both AI and AAF (Pearson's correlation coefficient: AI 0.86 , AAF $0.79, p<10^{-6}$ for both). However, while spontaneous rates in AAF showed the same depth dependence as the driven rates, in AI spontaneous rates appeared to peak at a shallower depth of $400 \mu \mathrm{m}$. Consistent with this observation, spontaneous rates normalized by the driven rate (Fig. $10 \mathrm{~B}$ ) showed no depth dependence in $\operatorname{AAF}(p>0.9$, Kruskal-Wallis test), but were significantly depth dependent in AI $(p<0.001$, Kruskal-Wallis test). Thus, the observed depth dependencies in spontaneous and 

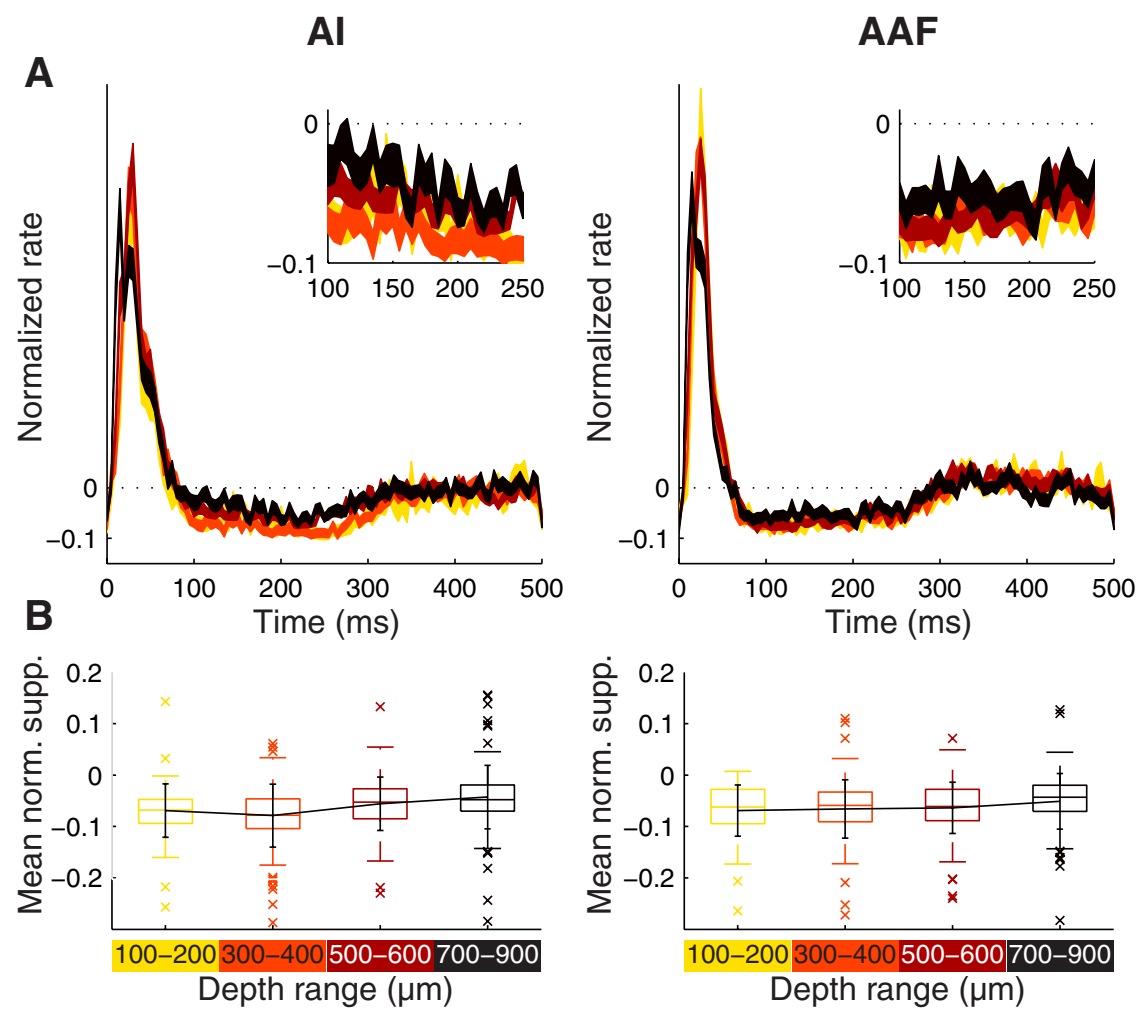

Figure 8. Magnitude of post-activation suppression is depth dependent. $\boldsymbol{A}$, Population PSTHs broken into coarse depth categories reveal that the post-activation suppression of activity is greater in superficial layers in AI (left), and to a lesser extent in AAF (right). Each cluster PSTH contributing to these population PSTHs was shifted to have a spontaneous rate of zero and then normalized to a maximum of one before averaging across clusters. Inset shows an expanded view of activity over the $100-250 \mathrm{~ms}$ poststimulus interval. Width of population PSTHs gives mean \pm SEM of all cluster PSTHs within the relevant depth range; lighter shades correspond to more superficial depths (color scheme as in $\boldsymbol{B}$ ). $\boldsymbol{B}$, Mean values of the normalized individual cluster PSTHs over the time range of $100-250 \mathrm{~ms}$, grouped into coarse depth categories as in $\boldsymbol{A}$. More negative values indicate deeper postactivation suppression. In Al, the suppression is greater in mid-to-superficial layers (Kruskal-Wallis test, $p<10^{-5}$; black lines give mean \pm SEM; colored lines in box give the median; colored box extends from the 25th to 75th percentile; whiskers show the extent of the data out to at most half again the interquantile distance; outliers are marked with $\mathrm{x}$ ). In AAF, the effect is weaker, but the suppression is significantly less in the deepest layer than in the most superficial layers (Kruskal-Wallis test, $p<0.01$ ).

driven firing rates cannot be explained by laminar variations that would be expected to be similar in two core auditory cortical areas (e.g., laminar differences in cell density or cell size).

\section{Discussion}

\section{Key findings}

We report here that cortical responses to slow trains of noise bursts vary systematically across depth in mouse auditory cortex. In particular, non-monotonic following occurs more frequently in mid-to-superficial than deep layers, and following declines at slower train rates in deep than superficial layers. These findings establish two key constraints on auditory cortical laminar computation: the existence of some mechanism generating nonmonotonic following in superficial layers, and the existence of a separate mechanism constraining propagation of following information from superficial to deep layers. Additionally, we find differences between cortical areas, with more non-monotonic following and better following at faster train rates in AAF than AI, supporting the idea that AAF is specialized for faster temporal processing (Linden et al., 2003).

We also report that post-activation suppression is stronger in superficial than deep layers, a finding that could account for some of the other depth dependencies we observe. The FI at the fastest train rate increased with depth, consistent with weaker post-activation suppression at deeper depths. Augmenting responses and non-monotonicity in FI-rate functions were strongest in more superficial layers and at stimulus rates of 3-4 bursts/s, suggesting that these responses are driven by some form of rebound from depth-dependent post-activation suppression. Also, the decrease in temporal precision of firing with increasing depth would be expected to arise from decreasing post-activation suppression of spontaneous activity. Previous studies have linked post-activation suppression with forward suppression (Phillips et al., 1989; Eggermont, 1992; Calford and Semple, 1995; Brosch and Schreiner, 1997), which is thought to reflect prolonged synaptic depression (Wehr and Zador, 2005). We therefore propose that a major laminar difference in auditory cortical responses is a decrease with depth in the magnitude of post-activation suppression, forward suppression, and possibly synaptic depression.

\section{Comparison with previous work}

We observed few qualitative differences in response properties between middle and more superficial layers of mouse auditory cortex. This result appears to contradict that of Atencio and Schreiner (2010), who found selectivity for faster temporal modulation rates in layer IV of cat auditory cortex than in superficial or deep layers. However, as they note, while their results are consistent with the pattern of rate selectivity observed in cat primary visual cortex (Hawken et al., 1996), studies in the squirrel primary visual cortex found fastest rate selectivity in the supragranular layers (Heimel et al., 2005). Thus, the divergent results may reflect a consistent species difference, perhaps arising from stronger recurrent connections between layer IV and layers II-III in rodent auditory cortex (Barbour and Callaway, 2008).

Most other previous studies of cortical entrainment to slow train rates have not pursued depth dependencies, but all have noted a pronounced decline in following as stimulation rates increase from 1 to 20 bursts/s (cat: Phillips et al., 1989; Eggermont, 1991, 1992; guinea pig: Creutzfeldt et al., 1980, rat: Kilgard and Merzenich, 1999; Anderson et al., 2006). Evidence for nonmonotonic following and augmenting responses also appears in some previous reports (Phillips et al., 1989; Eggermont, 1991, 1992; Kilgard and Merzenich, 1999). However, it is necessary to be cautious in drawing comparisons, because most studies have not directly assessed within-train burst responses relative to first-burst responses over a fixed response window. With other commonly used measures of cortical following, apparent nonmonotonic following or augmentation might not represent true facilitation of responses, but instead reflect sensitivity of following measures to stimulation frequency, spontaneous rate or postactivation suppression (see Eggermont, 1991 for discussion). The previous studies using measures most analogous to our following index were conducted in rat (Kilgard and Merzenich, 1999; bar- 
Al

A
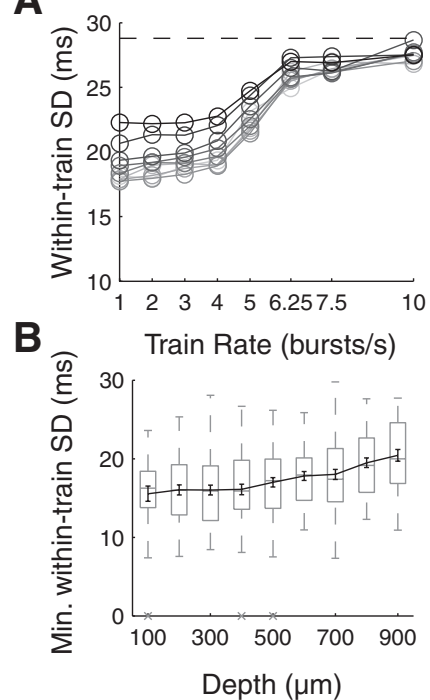

C

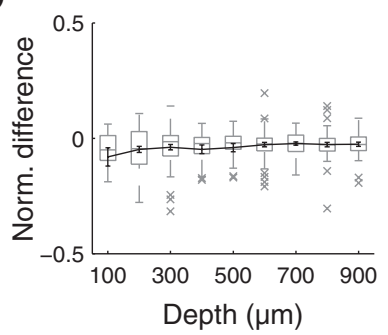

AAF
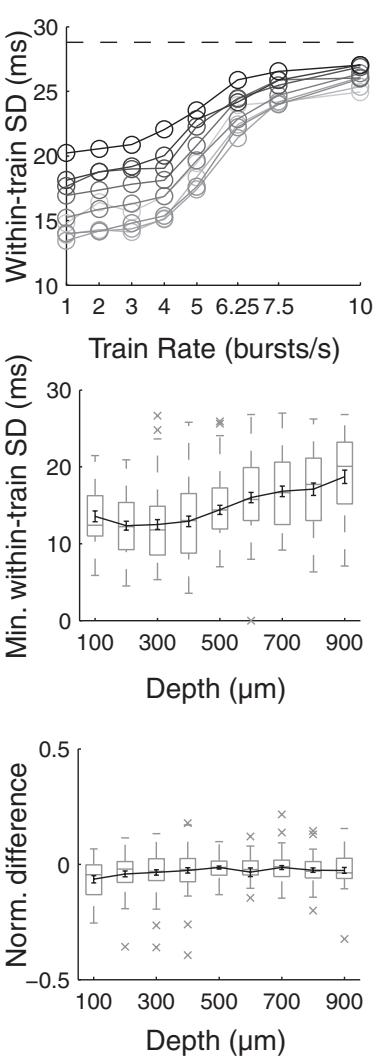

Figure 9. Precision of spike timing in responses to noise bursts is depth dependent in Al and AAF. A, Population averages of within-train spike-time SD as a function of train rate for Al (left) and AAF (right); each curve represents a different depth, with lighter gray indicating more superficial depths. Dotted line indicates the $28.8 \mathrm{~ms}$ SD expected if spikes were uniformly distributed over the analysis interval. Error bars are omitted for visual clarity. $\boldsymbol{B}$, The minimum within-train spike-time SD over train rates increases with depth in both Al and AAF (KruskalWallis test, $p<10^{-5}$; black lines give mean \pm SEM; gray lines in box indicate the median; box extends from the 25th to 75th percentile; whiskers show the extent of the data out to at most half again the interquantile distance; and outliers are marked with $\mathrm{x}$ ). $C$, The difference between first-burst spike-time SD and minimum within-train spike-time SD, normalized by the sum of the two SD, is near zero at all depths, and shows no significant depth dependence in either Al or AAF (Kruskal-Wallis test, $p>0.1$ ).

biturate anesthesia) and cat (Eggermont, 1991; Eggermont and Smith, 1995; ketamine anesthesia). Both found augmenting and rolloff in following at 8-10 bursts/s, compared with 3-4 bursts/s observed here in mouse, suggesting either species differences or effects of differences in anesthesia.

It is known that anesthesia can induce cortical oscillations, which might lead to non-monotonic following (Kenmochi and Eggermont, 1997). However, in our preparation, the dominant frequency of spontaneous oscillations in spiking was $2 \mathrm{~Hz}$ (data not shown), consistent with the frequency of oscillations observed in the mouse thalamic reticular nucleus in vitro (Warren et al., 1994). Since we found augmenting responses primarily at 3-4 bursts/s, the observed non-monotonic effects cannot be explained by a simple recruitment of anesthesia-induced oscillations, and must arise either from a separate mechanism entirely, or from a circuit whose "resonant frequency" changes in the presence of a driving stimulus. This is not to say that anesthesia played no role in our results, as ketamine-induced changes in auditory cortical response properties (Zurita et al., 1994; Gaese and Ostwald, 2001) could have either uncovered or masked augmenting responses.
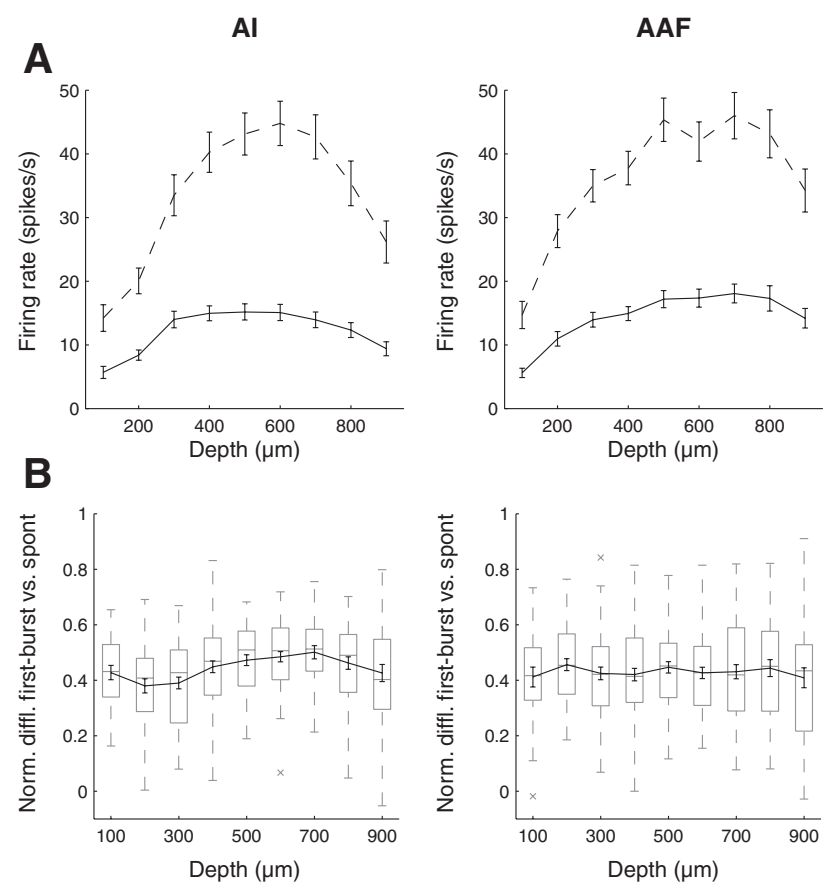

Figure 10. Spontaneous and driven firing rates are depth dependent. A, Both the spontaneous firing rate (solid line) and the mean firing rate in response to the first noise burst in all trains (dashed line) vary with depth in both Al (left) and AAF (right). Data are mean firing rates \pm SEM over all multiunit dusters recorded at each depth. Box plots are omitted for visual clarity since two datasets are plotted in the same axes. For both areas, the dependence on depth is significant (Kruskal-Wallis test, $p<10^{-6}$ ) with a peak at $\sim 600 \mu \mathrm{m}$. $\boldsymbol{B}$, The difference between first-burst response rate and spontaneous rate, normalized by the sum of the two rates, is constant relative to depth in AAF (right; Kruskal-Wallis test, $p>0.5$ ), but varies with depth in Al (left; Kruskal-Wallis test, $p<10^{-3}$ ). Black lines give mean \pm SEM; gray lines in box indicate the median; box extends from the 25th to 75th percentile; whiskers show the extent of the data out to at most half again the interquantile distance; and outliers are marked with $\mathrm{x}$.

It is possible that augmenting responses and non-monotonic following at 3-4 bursts/s reflect cortical specialization for ethologically significant sound modulation rates (Kim and Bao, 2009); however, there is no obvious correspondence with typical rates of mouse vocalizations (Liu et al., 2003). Rather, we suggest that the non-monotonic effects may reflect fundamental properties of thalamocortical and intracortical circuits. Augmentation of cortical evoked potentials during repeated electrical stimulation of the thalamus in vivo is a well known phenomenon (Dempsey and Morison, 1943), and maximal at mid-to-superficial depths in primary sensory cortices (Morin and Steriade, 1981; Ferster and Lindström, 1985; Metherate and Ashe, 1994). Similarly, almost all of the augmenting responses to auditory stimulation observed here were recorded at depths less than $500 \mu \mathrm{m}$, corresponding to layers I-IV in mouse (Anderson et al., 2009).

\section{Implications for cortical circuit function}

It is unlikely that differences in train-dependent response properties between AI and AAF, and between mid-to-superficial and deep layers, are inherited directly from the thalamus. Although there are differences in the patterns of input from the various thalamic subdivisions to AI and AAF (Huang and Winer, 2000), and to different cortical layers within each area (for review, see Linden and Schreiner, 2003), most neurons in all thalamic subdivisions reliably follow stimuli at much higher repetition rates than observed here for cortex (Creutzfeldt et al., 1980; Miller et al., 2002). Differences in following between AI and AAF could 
arise from different time constants of depression at thalamocortical synapses, but the existence of strong feedforward inputs from superficial to deep layers (Sakata and Harris, 2009) requires looking beyond the thalamocortical synapse to explain differences in following between mid-to-superficial and deep layers.

In particular, two key findings require explanation. First, augmenting responses and non-monotonic following are largely confined to mid-to-superficial layers, occur at train rates of 3-4 bursts/s, and are more pronounced in AAF than AI. This finding implies that mid-to-superficial-layer cortical circuits become transiently hyperexcitable $\sim 300 \mathrm{~ms}$ after a brief sensory input, especially in AAF. Such a transient increase in gain might arise from changes in membrane excitability. For instance, $G_{A B A}$ conductances persist for $200-300 \mathrm{~ms}$ in vivo in rat auditory cortex (Wehr and Zador, 2005), and the associated hyperpolarization might activate hyperpolarization-sensitive cation channels, leading to rebound excitation. Elements of such a mechanism are already known to be layer-specific (e.g., in mouse auditory cortex: Huggenberger et al., 2009).

Alternatively, hyperexcitability might arise at the circuit level, from differing durations of depression at different types of synapse. In mid-to-superficial layers, excitatory intracortical synapses may recover more quickly than inhibitory synapses (Metherate and Ashe, 1994; Wehr and Zador, 2005), leading to transient shifts in the excitation-inhibition balance of the recurrent circuit (Houweling et al., 2002). Or, both types of intracortical synapse might recover from depression more rapidly than do thalamocortical synapses. If intracortical synapses normally operate in a depressed baseline state due to spontaneous thalamocortical input, release from this depression could generate hyperexcitability (cf. Loebel et al., 2007).

The second key finding is that, despite the existence of strong feedforward input from superficial to deep layers (Sakata and Harris, 2009), some stimulus rates that are highly effective in driving mid-to-superficial layers elicit poor following in deeper layers. This result cannot be explained by lower excitability in the infragranular network; activity was consistently stronger in deep than superficial layers. Instead, the earlier rolloff in following and reduction in non-monotonic following in deep layers requires some mechanism for decoupling superficial from deep layers. One possibility is that only superficial-layer neurons with early rolloff and relatively monotonic rate-following functions project to deeper layers, and the remainder of superficial-layer units are involved in other circuits.

Another possibility is that decoupling could be introduced via depression of feedforward synapses from superficial layers. Neurons in deep layers could remain relatively active despite decoupling from feedforward superficial layer input, through recurrent intracortical excitation and nonprimary thalamic input; previous work has shown that during silence, spontaneous activity waves in cortex often originate in deep rather than mid-to-superficial layers (Sakata and Harris, 2009). The cortical network might operate in the "sensory-evoked" feedforward mode of information flow from superficial to deep layers only within the first few tens of milliseconds after a brief sensory input (cf. Curto et al., 2009); depression of feedforward intracortical synapses might then decouple the deep layers from superficial layers to generate activity primarily through other sources, until the feedforward synapses from superficial layers recovered after an interval $(>300-350$ $\mathrm{ms}$ ) sufficient to reduce following over the relevant timescale.

In conclusion, our results not only demonstrate laminar differences in temporal processing over slow time scales in auditory cortex, but also lead to the following predictions about auditory cortical circuitry: (1) cellular or circuit mechanisms drive transient increases in gain in mid-to-superficial layers following postactivation suppression; (2) interlaminar circuitry buffers these increases in gain and prevents them from propagating to deeper layers.

\section{References}

Abeles M, Goldstein MH (1970) Functional architecture in cat primary auditory cortex: columnar organization and organization according to depth. J Neurophysiol 33: 172-187.

Ahissar E, Sosnik R, Bagdasarian K, Haidarliu S (2001) Temporal frequency of whisker movement. II. Laminar organization of cortical representations. J Neurophysiol 86:354-367.

Anderson LA, Christianson GB, Linden JF (2009) Mouse auditory cortex differs from visual and somatosensory cortices in the laminar distribution of cytochrome oxidase and acetylcholinesterase. Brain Res 1252:130-142.

Anderson SE, Kilgard MP, Sloan AM, Rennaker RL (2006) Response to broadband repetitive stimuli in auditory cortex of the unanesthetized rat. Hear Res 213:107-117.

Atencio CA, Schreiner CE (2010) Laminar diversity of dynamic sound processing in cat primary auditory cortex. J Neurophysiol 103:192-205.

Atencio CA, Sharpee TO, Schreiner CE (2009) Hierarchical computation in the canonical auditory cortical circuit. Proc Natl Acad Sci U S A 106:21894-21899.

Barbour DL, Callaway EM (2008) Excitatory local connections of superficial neurons in rat auditory cortex. J Neurosci 28:11174-11185.

Brosch M, Schreiner CE (1997) Time course of forward masking tuning curves in cat primary auditory cortex. J Neurophysiol 77:923-943.

Brumberg JC, Pinto DJ, Simons DJ (1999) Cortical columnar processing in the rat whisker-to-barrel system. J Neurophysiol 82:1808-1817.

Calford MB, Semple MN (1995) Monaural inhibition in cat auditory cortex. J Neurophysiol 73:1876-1891.

Creutzfeldt O, Hellweg FC, Schreiner C (1980) Thalamocortical transformation of responses to complex auditory stimuli. Exp Brain Res 39:87-104.

Curto C, Sakata. S, Marguet S, Itskov V, Harris KD (2009) A simple model of cortical dynamics explains variability and state dependence of sensory responses in urethane-anesthetized auditory cortex. J Neurosci 29:10600-10612.

deFelipe J, Jones EG (Eds.) (1988) Cajal on the Cerebral Cortex: An Annotated Translation of the Complete Writings. New York: Oxford UP.

Dempsey EW, Morison RS (1943) The electrical activity of a thalamocortical relay system. Am J Physiol 138:283-296.

Douglas RJ, Martin KAC (2004) Neuronal circuits of the neocortex. Annu Rev Neurosci 27:419-451.

Eggermont JJ (1991) Rate and synchronization measures of periodicity coding in cat primary auditory cortex. Hear Res 56:153-167.

Eggermont JJ (1992) Stimulus-induced and spontaneous rhythmic firing of single units in cat primary auditory cortex. Hear Res 61:1-11.

Eggermont JJ, Smith GM (1995) Synchrony between single-unit, activity and local field potentials in relation to periodicity coding in primary auditory cortex. J Neurophysiol 73:227-245.

Ferster D, Lindström S (1985) Augmenting responses evoked in area 17 of the cat by intracortical axon collaterals of cortico-geniculate cells. J Physiol 367:217-232.

Gaese BH, Ostwald J (2001) Anesthesia changes frequency tuning of neurons in the rat primary auditory cortex. J Neurophysiol 86:1062-1066.

Gilbert. CD (1977) Laminar differences in receptive field properties of cells in cat primary visual cortex. J Physiol 268:391-421.

Goldberg JM, Brown PB (1969) Response of binaural neurons of dog superior olivary complex to dichotic tonal stimuli: some physiological mechanisms for sound localization. J Neurophysiol 32:613-636.

Hawken MJ, Shapley RM, Grosof DH (1996) Temporal-frequency selectivity in monkey visual cortex. Vis Neurosci 13:477-492.

Heimel JA, Hooser SDY, Nelson SB (2005) Laminar organization of response properties in primary visual cortex of the gray squirrel (Sciurus carolinensis). J Neurophysiol 94:3538-3554.

Houweling AR, Bazhenov M, Timofeev I, Grenier F, Steriade M, Sejnowski T] (2002) Frequency-selective augmenting responses by short-term synaptic depression in cat neocortex. J Physiol 542:599-617.

Huang CL, Winer JA (2000) Auditory thalamocortical projections in the cat: laminar and area! patterns of input. J Comp Neurol 427:302-331. 
Hubel DH, Wiesel TN (1962) Receptive fields, binocular interaction and functional architecture in the cat's visual cortex. J Physiol 160:106-154.

Huggenberger S, Vater M, Deisz RA (2009) Interlaminar differences of intrinsic properties of pyramidal neurons in the auditory cortex of mice. Cereb Cortex 19:1008-1018.

Kenmochi M, Eggermont JJ (1997) Autonomous cortical rhythms affect temporal modulation transfer functions. Neuroreport 8:1589-1593.

Kilgard MP, Merzenich MM (1999) Distributed representation of spectral and temporal information in rat primary auditory cortex. Hear Res 134:16-28.

Kim H, Bao S (2009) Selective increase in representations of sounds repeated at an ethological rate. J Neurosci 29:5163-5169.

King A J, Nelken I (2009) Unraveling the principles of auditory cortical processing: can we learn from the visual system? Nat Neurosci 12: $698-701$.

Kral A, Hartmann R, Tillein J, Heid S, Klinke R (2000) Congenital auditory deprivation reduces synaptic activity within the auditory cortex in a layerspecific manner. Cereb Cortex 10:714-726.

Linden JF, Schreiner CE (2003) Columnar transformations in auditory cortex? A comparison to visual and somatosensory cortices. Cereb Cortex 13:83-89.

Linden JF, Liu RC, Sahani M, Schreiner CE, Merzenich MM (2003) Spectrotemporal structure of receptive fields in areas AI and AAF of mouse auditory cortex. J Neurophysiol 90: 2660-2675.

Liu RC, Miller KD, Merzenich MM, Schreiner CE (2003) Acoustic variability and distinguishability among mouse ultrasound vocalizations. J Acoust Soc Am 114: 3412-3422.

Loebel A, Nelken I, Tsodyks M (2007) Processing of sounds by population spikes in a model of primary auditory cortex. Front Neurosci 1: 197-209.

Mendelson JR, Schreiner CE, Sutter ML (1997) Functional topography of cat primary auditory cortex: response latencies. J Comp Physiol A Neuroethol Sens Neural Behav Physiol 181: 615-633.

Metherate R, Ashe JH (1994) Facilitation of an NMDA receptor-mediated EPSP by paired-pulse stimulation in rat neocortex via depression of GABAergic IPSPs. J Physiol 481: 331-348.

Miller LM, Escabí MA, Read HL, Schreiner CE (2002) Spectrotemporal receptive fields in the lemniscal auditory thalamus and cortex. J Neurophysiol 87: 516-527.
Morin D, Steriade M (1981) Development from primary to augmenting responses in the somatosensory system. Brain Res 205: 49-66.

Nelken I, Fishbach A, Las L, Ulanovsky N, Farkas D (2003) Primary auditory cortex of cats: feature detection or something else? Biol Cybern 89: 397-406.

Phillips DP, Irvine DR (1981) Responses of single neurons in physiologically defined primary auditory cortex (AI) of the cat: frequency tuning and responses to intensity. J Neurophysiol 45: 48-58.

Phillips DP, Hall SE, Hollett JL (1989) Repetition rate and signal level effects on neuronal responses to brief tone pulses in cat auditory cortex. J Acoust Soc Am 85: 2537-2549.

Sahani M (1999) Latent variable models for neural data analysis. Ph.D. thesis, California Institute of Technology.

Sakata S, Harris KD (2009) Laminar structure of spontaneous and sensoryevoked population activity in auditory cortex. Neuron 64: 404-418.

Shen JX, Xu ZM, Yao YD (1999) Evidence for columnar organization in the auditory cortex of the mouse. Hear Res 137: 174-177.

Stiebler I, Neulist R, Fichtel I, Ehret G (1997) The auditory cortex of the house mouse: left-right differences, tonotopic organization and quantitative analysis of frequency representation. J Comp Physiol 181: 559-571.

Sugimoto S, Sakurada M, Horikawa J, Taniguchi I (1997) The columnar and layer-specific response properties of neurons in the primary auditory cortex of Mongolian gerbils. Hear Res 112: 175-185.

Ulanovsky N, Las L, Farkas D, Nelken I (2004) Multiple time scales of adaptation in auditory cortex neurons. J Neurosci 24: 10440-10453.

Wallace MN, Palmer AR (2008) Laminar differences in the response properties of cells in the primary auditory cortex. Exp Brain Res 184: 179-191.

Warren RA, Agmon A, Jones EG (1994) Oscillatory synaptic interactions between ventroposterior and reticular neurons in mouse thalamus in vitro. J Neurophysiol 72: 1993-2003.

Wehr M, Zador AM (2005) Synaptic mechanisms of forward suppression in rat auditory cortex. Neuron 47: 437-445.

Zheng QY, Johnson KR, Erway LC (1999) Assessment of hearing in 80 inbred strains of mice by ABR threshold analyses. Hear Res 130: 94-107.

Zurita P, Villa AE, de Ribaupierre Y, de Ribaupierre F, Rouiller EM (1994) Changes of single unit activity in the cat's auditory thalamus and cortex associated to different anesthetic conditions. Neurosci Res 19: 303-316. 